\title{
Novel real-time PCR for the universal detection of Strongyloides species
}

\author{
Correspondence \\ Stefanie Kramme \\ stefanie.kramme@unibas.ch \\ Norbert Brattig \\ nbrattig@bni-hamburg.de
}

Received 18 August 2010

Accepted 15 December 2010

\author{
Stefanie Kramme,† Nicole Nissen, Hanns Soblik, Klaus Erttmann, \\ Egbert Tannich, Bernhard Fleischer, Marcus Panning and Norbert Brattig
}

Bernhard Nocht Institute for Tropical Medicine, Hamburg, Germany

\begin{abstract}
Strongyloidiasis is a neglected disease that is prevalent mainly in tropical and subtropical regions. It is caused by intestinal nematodes of the genus Strongyloides. Due to the rise in worldwide travel, infections are increasingly encountered in non-endemic regions. Diagnosis is hampered by insensitive and laborious detection methods. A universal Strongyloides species real-time PCR was developed with an internal competitive control system. The $95 \%$ limit of detection as determined by probit analysis was one larva per PCR equivalent to 100 larvae per $200 \mathrm{mg}$ stool. The assay proved to be $100 \%$ specific as assessed using a panel of parasites and bacteria and thus might be useful in the diagnostic setting as well as for Strongyloides research.
\end{abstract}

\section{INTRODUCTION}

Nematodes of the genus Strongyloides are widely distributed in tropical and subtropical countries and pose a significant health threat to humans (Bethony et al., 2006). An estimated 30-100 million people are infected worldwide. In light of frequent international travel and immigration, strongyloidiasis is increasingly encountered in travel clinics in nonendemic regions (Nuesch et al., 2005). At least two species are known to infect humans, Strongyloides stercoralis and Strongyloides fuelleborni. Strongyloides ratti, which naturally occurs in rats, is widely used as an experimental infection model to study strongyloidiasis (Viney, 1999). In immunocompetent humans, Strongyloides infection usually remains asymptomatic or is associated with mild and nonspecific symptoms only (Olsen et al., 2009). Due to the ability to replicate within the host, chronic auto-infection may occur and long-term persistence of Strongyloides infection over decades has been described (Igual-Adell et al., 2004). In immunocompromised hosts, however, S. stercoralis infections usually present as severe hyperinfection syndromes with mortality rates of up to $87 \%$ (Marcos et al., 2008).

Standard diagnostic procedures in humans comprise concentration and stool culture methods (Siddiqui \& Berk, 2001). A combination of these methods yields highest sensitivity especially if multiple stool samples are examined. However, this procedure is rather laborious and timeconsuming. Several serum antibody detection methods do exist with variable sensitivities and specificities depending on the antigen used (Ravi et al., 2002), the immunoglobulin isotypes or the study population (Silva et al., 2003;

tPresent address: Swiss Tropical and Public Health Institute, Socinstrasse 57, CH-4002 Basel, Switzerland.

Abbreviations: FRET, fluorescence energy transfer; LOD, limit of detection.
Rodrigues et al., 2007). As a major drawback of serological tests, considerable cross-reactivity between the various nematode species has been observed. Additionally, antibody detection methods lack sensitivity especially in returning travellers (Sudarshi et al., 2003).

As DNA detection becomes increasingly utilized in the diagnosis of parasite infection, this method might have the potential to overcome the problems in diagnosing strongyloidiasis (ten Hove et al., 2009). A novel real-time PCR detection system recently proved successful in a small pilot study but data on its analytical sensitivity are lacking (Verweij et al., 2009). In this communication, we report on the development of a real-time PCR assay for sensitive detection of Strongyloides species DNA in stool samples which can be used both in research and in the diagnostic setting. The assay was thoroughly evaluated from a technical point of view. In addition, an internal control system was established to monitor the influence of possible inhibitory activity.

\section{METHODS}

Parasite isolates. The $S$. ratti infection cycle established in our laboratory was originally provided by Dr G. Pluschke (Swiss Tropical Institute, Basel). Wistar rats were used to maintain the cycle by serial passage as described by Viney \& Lok (2007). In brief, Wistar rats were infected subcutaneously with 2500 L3 S. ratti larvae. Faeces were collected from day 1 until day 40. Approval was obtained from the animal protection board of the City of Hamburg. All faecal pellets were collected daily from day 1 until day 40, suspended in PBS with charcoal and incubated for 5 days at $26{ }^{\circ} \mathrm{C}$ (Viney \& Lok, 2007). Infective larvae were collected by the standard Baermann extraction technique (García \& Bruckner, 2001), centrifuged, resuspended and counted microscopically.

DNA preparation from stool samples. For isolation of nucleic acids from stool samples, the QIAmp DNA stool kit (Qiagen) was 
used according to the manufacturer's instructions. Two hundred milligrams of human stool or faeces from infected as well as uninfected rats was used.

Real-time PCR. A novel real-time PCR for Strongyloides species targeting the $28 \mathrm{~S}$ rRNA gene was developed (Fig. 1). Using BLAST search software (http://ncbi.nlm.nih.gov/BLAST/), all available Strongyloides species $28 \mathrm{~S} \mathrm{rRNA}$ sequences were aligned. Upon manual inspection, a conserved region was identified fitting the demands of fluorescence energy transfer (FRET)-based real-time PCR assays. A $20 \mu \mathrm{l}$ reaction contained $2 \mu \mathrm{l} \mathrm{PCR}$ buffer (Applied Biosystems), $200 \mu \mathrm{M}$ of each dNTP (Applied Biosystems), $2.5 \mathrm{mM} \mathrm{MgCl} 2,750 \mathrm{nM}$ StroS (5'-TTAGAGTCGTGTTGCTTGGAA- $3^{\prime}$ ), $1 \mu \mathrm{M}$ StroAS (5'-GTGCAACTGGCTCTGTATGC-3'), $50 \mathrm{nM}$ StroP3 (5'-CTGTGAAGGAAAATTGCAAAGTACTCCGGA-FAM-3'), 200 nM StroP4 (5'-LC640-GAGAGTTAAAGAGGACGTGAAACCGATACG-PH-3'), $200 \mathrm{nM}$ StroP5 (5' LC705-ATCGTTCGTTGAGCGATTAGCAGTT-PH-3'), $0.8 \mu \mathrm{g}$ BSA and $1 \mathrm{U}$ AmpliTaq Gold DNA polymerase (Applied Biosystems). The amplified fragment length was $180 \mathrm{bp}$. Probe StroP3 was labelled with fluorescein at its $3^{\prime}$-end and probe StroP4 was labelled with LightCycler Red 640 at its $5^{\prime}$-end and phosphorylated at the $3^{\prime}$-end. Correspondingly, StroP5 was labelled with LightCycler Red 705 at its $5^{\prime}$-end and phosphorylated at the $3^{\prime}$-end. Two microlitres of DNA solution was added to each reaction. Thermal cycling in a LightCycler 1.5 Instrument (Roche Diagnostics) involved denaturation at $95{ }^{\circ} \mathrm{C}$ for $15 \mathrm{~min}$, followed by 45 cycles of $95{ }^{\circ} \mathrm{C}$ for $5 \mathrm{~s}, 50{ }^{\circ} \mathrm{C}$ for $20 \mathrm{~s}$ and $72{ }^{\circ} \mathrm{C}$ for $30 \mathrm{~s}$. Fluorescence was measured during the $50{ }^{\circ} \mathrm{C}$ step on detection channel F2 $(640 \mathrm{~nm})$ and divided by fluorescence measured on channel F1 $(530 \mathrm{~nm})$ for normalization. The internal control signal was measured in channel F3 $(705 \mathrm{~nm})$ of the LightCycler instrument and divided by fluorescence measured on channel F1 $(530 \mathrm{~nm})$.

Construction of DNA standard. The $180 \mathrm{bp}$ real-time amplicon of the $28 \mathrm{~S}$ rRNA gene of $S$. ratti was ligated into pCR2.1 plasmid vector and cloned into Escherichia coli using the pCR 2.1 TOPO TA cloning kit (Invitrogen) as described by Panning et al. (2008). Resulting plasmids (pStrowild) of the correct length were purified by means of a QIAprep Spin Miniprep kit (Qiagen) and DNA concentration was spectrophotometrically measured. Sequence integrity was checked by sequencing on a CEQ 8000 Genetic Analysis System (Beckmann Coulter).

Construction of internal control. A competitive internal control system was incorporated into the assay by gene splicing and overlap extension as described by Panning et al. (2008). In brief, using primers IcS (5' -ATCGTTCGTTGAGCGATTAGCAGTTGTTATGGAAGCATACAGAGCCAGTTGCAC-3') and IcAs (5'-AACTGCTAATCGCTCAACGAACGATTCTCCGGAGTACTTTGCAATTTTCCTTC$3^{\prime}$ ), an alternative probe binding site was introduced into pStrowild yielding pStroIC. A probe alternatively labelled with dye LC705 (StroP5) was used for detection of pStroIC in a separate channel of the LightCycler instrument.

Probit analysis. The lower limit of detection (LOD) was determined by probit analysis of multiple parallel amplifications $(\times 5)$ of stool spiked with plasmid pStrowild as described by Panning et al. (2008). In addition, different input numbers of microscopically determined $S$. ratti larvae were spiked in human stool samples from healthy German volunteers which tested negative for parasites upon microscopy. All samples were correspondingly tested in multiple parallel amplifications. Statgraphics Plus, version 5 (Manugistics), was used for statistical analysis.

Cross-reactivity panel. DNA from the following parasites was tested with the novel real-time PCR: Ancylostoma duodenale, Necator americanus, Trichuris trichiura, Ascaris suum, Trichinella spiralis, Toxocara canis, Wuchereria bancrofti, Loa loa, Dirofilaria immitis, 
(a)

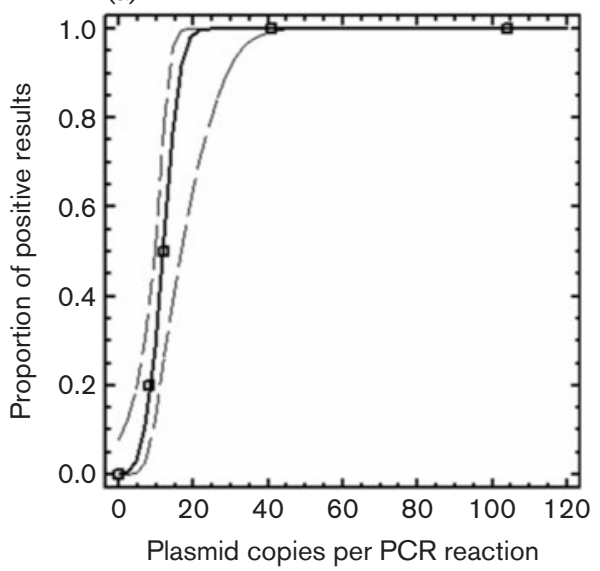

(b)

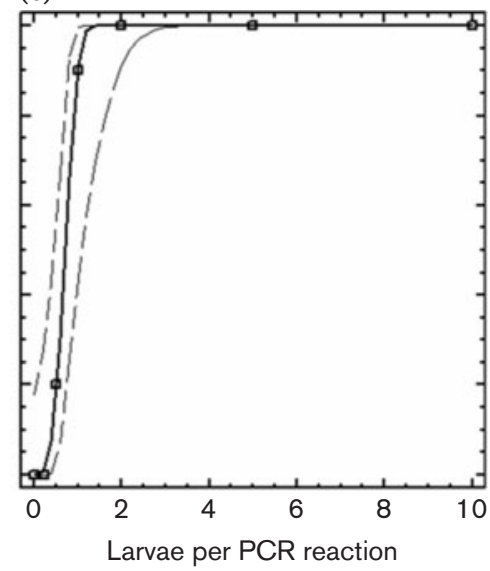

Fig. 2. Probability of achieving a positive test result ( $y$-axis), depending on DNA copy input number per PCR ( $x$-axis). Each datum point represents the rate of positive results in five replicate tests per concentration. Broken lines indicate $95 \%$ confidence intervals. (a) Plasmid pStrowild spiked in human stool samples; (b) microscopically counted larvae spiked in human stool samples.
Echinococcus granulosus and Schistosoma mansoni. In addition and to test for possible cross-reactivity, nucleic acid samples from the following panel of common bacteria and viruses were used (Panning et al., 2007): Epstein-Barr virus (patient isolate), hepatitis B virus (INSTAND ref. material 11019), hepatitis C virus (1st International WHO NAT standard), human cytomegalovirus (INSTAND ref. material 15005), herpes simplex virus type 1 (INSTAND ref. material 13017), human immunodeficiency virus type 1 (NL-43), E. coli (ATCC 25922), Pseudomonas aeruginosa (in-house reference strain), Staphylococcus aureus (ATCC 13565) and Streptococcus pyogenes (ATCC 19615). In addition, DNA from the protozoan parasite Plasmodium falciparum (patient isolate) was used.

Clinical specimens. Thirty-five frozen stool samples from patients visiting the outpatient clinic of the Bernhard Nocht Institute for Tropical Medicine for reasons other than suspected strongyloidiasis were analysed retrospectively. All stool samples were negative for parasites upon microscopic inspection. Concomitantly, ten stool samples from ten patients with microscopic detection of Entamoeba dispar cysts, ten patients with Giardia lamblia and three patients with Enterobius species eggs as well as one patient positive for Ascaris suum and one patient with hookworms, were tested. Residual testing of patient samples was approved by the institutional review board of the Bernhard Nocht Institute.

\section{RESULTS AND DISCUSSION}

A novel real-time PCR using FRET technology was designed targeting the $28 \mathrm{~S}$ rRNA gene of Strongyloides species. The assay was set up allowing the simultaneous detection of S. stercoralis, S. fuelleborni, S. ratti, Strongyloides robustus, Strongyloides calloscinreus and Strongyloides procyonis. To date, only S. stercoralis and S. fuelleborni are known to cause infection in humans but infection with other species might be possible. In addition, we aimed to provide a universal detection tool that can also be used in the $S$. ratti infection model.

\section{Analytical sensitivity}

Initial experiments were done with extracted DNA from $S$. ratti as well as $S$. stercoralis to optimize the assay with respect to reaction conditions. Following optimization, a plasmid,
pStrowild, was constructed and used to exactly determine the analytical sensitivity of the assay. Five copies per PCR of plasmid pStrowild were regularly detectable, indicating a high analytical sensitivity of the assay. A linear relationship between the real-time PCR crossing point and the $\log _{10}$ input DNA copy number was observed over a wide range of concentrations $\left(10^{6}-10^{2}\right.$ DNA copies per reaction).

An internal competitive control was incorporated into the assay. As single copies of pStroIC were detectable, this approach circumvents the need for multiplex PCRs, which in general can reduce sensitivity and reliability of assays. To exclude the possibility that the internal control affected the amplification efficiency of Strongyloides species detection, 20 copies of pStroIC per reaction were chosen as the working concentration for all further experiments. This working concentration did not reduce the overall sensitivity of the assay (data not shown) and has already been successfully incorporated into PCR assays using competitive internal controls (Panning et al., 2007, 2008). In further experiments, the internal control was spiked into the lysis buffer and was co-purified with the sample. Crosstalk into the wild-type channel was not observed.

The $95 \%$ LOD was determined next. This common technical specification indicates the concentration down to which an assay will detect the analyte with at least $95 \%$ probability. In order to mimic the clinical situation, human stool specimens were spiked with plasmid pStrowild and served as reference material. Different concentrations of DNA were extracted as described above. Replicate testing of each analyte concentration yielded a $95 \%$ LOD of 18 DNA copies per PCR [95\% confidence interval (CI) 14-32 DNA copies per PCR] equivalent to 1800 DNA copies per $200 \mathrm{mg}$ stool (95\% CI 1400-3200 DNA copies per $200 \mathrm{mg}$ stool) (Fig. 2). The internal control yielded a valid signal in every reaction, indicating the robustness of the assay. As stool samples may contain a number of substances that are able to inhibit PCRs, an internal competitive control was introduced, which offered the opportunity to monitor for inhibitory activity throughout the whole extraction as well as amplification process. Furthermore, the use of a spin 
column format to extract nucleic acids from stool samples has demonstrated its reliability in recent studies (Verweij et al., 2000; Blessmann et al., 2002). Next we determined the $95 \%$ LOD using microscopically counted S. ratti larvae spiked into human stool suspensions as described above. Replicate testing yielded a $95 \%$ LOD of of one larva per PCR (95\% CI 0.8-1.5 larvae per PCR) equivalent to 100 larvae per $200 \mathrm{mg}$ stool (95\% CI 80-150 larvae per $200 \mathrm{mg}$ stool) (Fig. 2). This LOD is comparable to that of published real-time PCR assays (Panning et al., 2008).

\section{Specificity}

To ensure a high specificity of the assay, FRET technology was applied, which relies on the concurrent binding of two probes in order to receive a fluorescence signal. This approach was chosen because of the sequence similarity within the group of nematodes. In contrast to a recent publication (Verweij et al., 2009) in which one Taqmanminor groove binder probe for amplicon detection was utilized, FRET technology might further reduce non-specific binding. Specificity of this assay was determined by testing various DNA preparations derived from different helminth species, in particular nematodes, without yielding positive amplification results with material other than Strongyloides species. Cross-reactivity was also tested on a panel of common bacteria and viruses with negative results.

Additionally, 35 stool extracts from symptomatic patients without microscopic detection of parasites yielded negative results upon testing by the novel real-time PCR. To exclude false positivity, 23 DNA stool preparations from patients with other microscopically confirmed parasitic diseases were tested without yielding positive results with the Strongyloides species assay. This finding is in concordance with Verweij et al. (2009), indicating the high specificity of the novel assay. Due to a lack of DNA from S. fuelleborni, $S$. robustus, S. calloscinreus and S. procyonis, we could not confirm the specificity of the assay for these pathogens. However, based on available sequence information, it is to be expected that these Strongyloides species will also be detectable with similar sensitivity (Fig. 1).

\section{Clinical applicability}

Clinical applicability of this assay could be demonstrated on five human stool samples with microscopic demonstration of $S$. stercoralis larvae. All specimens yielded a positive result upon use of the novel real-time PCR and subsequent sequencing of a fragment of the $28 \mathrm{~S}$ rRNA gene confirmed the presence of $S$. stercoralis DNA. Recently, a real-time PCR has been evaluated on a panel of patients rendering it a useful tool for diagnostics (Verweij et al., 2009). Compared to culture-based methods, it is suggested that real-time PCR can assist in the rapid diagnosis of strongyloidiasis especially when increasing numbers of patients have to be analysed (Blessmann et al., 2002). However, it should be noted that the sample input volume of different methods significantly influences diagnostic yield as already speculated (Verweij et al., 2009). In this respect, larger studies are mandatory to compare classical and molecular methods in detail. In addition, as a future prospect in endemic countries, more inexpensive methods are desirable.

\section{Application in an animal model}

Finally, a well-established rat model for $S$. ratti was used to further test the applicability of the assay. Rats were infected subcutaneously with infectious stage 3 larvae (iL3) of $S$. ratti. Stool samples were analysed by microscopy as well as by real-time PCR from day 0 to day 40 post-infection (p.i.). Each sample was tested in duplicate. Real-time PCR yielded positive results from day 4 p.i. onwards (Table 1), whereas microscopy became positive for the detection of first-stage larvae on day 5 p.i. PCR remained positive until day 22 whereas microscopy allowed larval detection until day 18 only. Although real-time PCR was found to be more sensitive, further studies are needed to compare the outcome of microscopy and real-time PCR. The target region, the 28S rRNA sequence, exists in various copies. Even though the exact copy number is not known, the probability of detection should be increased compared to that of a single copy gene. The concept of targeting multicopy genes has already been successfully used for other pathogens (Panning et al., 2008).

\section{Conclusion}

We demonstrated that this novel real-time PCR method can serve as a useful tool in both the clinical as well as a research setting. Analytical sensitivity and specificity are high and the assay meets today's standards of modern molecular diagnostic tools, i.e. an internal control system and quantification capabilities. It may thus assist decision making and therapy follow-up in the routine diagnostic laboratory as well as in research settings.

Table 1. Results of microscopy and real-time PCR within 23 days post-infection (p.i.) of Wistar rats with infective larvae

Each stool sample was analysed in duplicate. +, Positive result; -, negative result.

\begin{tabular}{|lcc|}
\hline Result days p.i. & Real-time PCR & Microscopy \\
\hline Day 0 & $-/-$ & $-/-$ \\
Day 1 & $-/-$ & $-/-$ \\
Day 2 & $-/-$ & $-/-$ \\
Day 3 & $-/-$ & $-/-$ \\
Day 4 & $+/+$ & $-/-$ \\
Day 5 & $+/+$ & $+/+$ \\
Days 6-18 & $+/+$ & $+/+$ \\
Days 19-22 & $+/+$ & $-/-$ \\
Day 23 & $-/-$ & $-/-$ \\
\hline
\end{tabular}




\section{ACKNOWLEDGEMENTS}

We greatly appreciate the excellent technical work of Kerstin Krausz. We are grateful to Dr G. Pluschke (Swiss Tropical Institute, Basel, Switzerland) for providing the $S$. ratti infection cycle. This study was supported by Vereinigung der Freunde des Tropeninstituts Hamburg.

\section{REFERENCES}

Bethony, J., Brooker, S., Albonico, M., Geiger, S. M., Loukas, A., Diemert, D. \& Hotez, P. J. (2006). Soil-transmitted helminth infections: ascariasis, trichuriasis, and hookworm. Lancet 367, 15211532 .

Blessmann, J., Buss, H., Nu, P. A., Dinh, B. T., Ngo, Q. T., Van, A. L., Alla, M. D., Jackson, T. F., Ravdin, J. I. \& Tannich, E. (2002). Real-time PCR for detection and differentiation of Entamoeba histolytica and Entamoeba dispar in fecal samples. J Clin Microbiol 40, 4413-4417.

García, L. S. \& Bruckner, D. A. (2001). Diagnostic Medical Parasitology. Washington, DC: American Society for Microbiology.

Igual-Adell, R., Oltra-Alcaraz, C., Soler-Company, E., SánchezSánchez, P., Matogo-Oyana, J. \& Rodríguez-Calabuig, D. (2004). Efficacy and safety of ivermectin and thiabendazole in the treatment of strongyloidiasis. Expert Opin Pharmacother 5, 2615-2619.

Marcos, L. A., Terashima, A., Dupont, H. L. \& Gotuzzo, E. (2008). Strongyloides hyperinfection syndrome: an emerging global infectious disease. Trans R Soc Trop Med Hyg 102, 314-318.

Nuesch, R., Zimmerli, L., Stockli, R., Gyr, N. \& Hatz, C. F. R. (2005), Imported strongyloidosis: a longitudinal analysis of 31 cases. J Travel Med 12, 80-84.

Olsen, A., van Lieshout, L., Marti, H., Polderman, T., Polman, K., Steinmann, P., Stothard, R., Thybo, S., Verweij, J. J. \& Magnussen, P. (2009). Strongyloidiasis - the most neglected of the neglected tropical diseases? Trans $R$ Soc Trop Med Hyg 103, 967-972.

Panning, M., Kramme, S., Petersen, N. \& Drosten, C. (2007). High throughput screening for spores and vegetative forms of pathogenic B. anthracis by an internally controlled real-time PCR assay with automated DNA preparation. Med Microbiol Immunol (Berl) 196, 4150.

Panning, M., Kilwinski, J., Greiner-Fischer, S., Peters, M., Kramme, S. Frangoulidis, D., Meyer, H., Henning, K. \& Drosten, C. (2008). High throughput detection of Coxiella burnetii by real-time PCR with internal control system and automated DNA preparation. $B M C$ Microbiol 8, 77.

Ravi, V., Ramachandran, S., Thompson, R. W., Andersen, J. F. \& Neva, F. A. (2002). Characterization of a recombinant immunodiagnostic antigen (NIE) from Strongyloides stercoralis L3-stage larvae. Mol Biochem Parasitol 125, 73-81.

Rodrigues, R. M., de Oliveira, M. C., Sopelete, M. C., Silva, D. A., Campos, D. M., Taketomi, E. A. \& Costa-Cruz, J. M. (2007). IgG1, IgG4, and IgE antibody responses in human strongyloidiasis by ELISA using Strongyloides ratti saline extract as heterologous antigen. Parasitol Res 101, 1209-1214.

Siddiqui, A. A. \& Berk, S. L. (2001). Diagnosis of Strongyloides stercoralis infection. Clin Infect Dis 33, 1040-1047.

Silva, L. P., Barcelos, I. S., Passos-Lima, A. B., Espindola, F. S., Campos, D. M. \& Costa-Cruz, J. M. (2003). Western blotting using Strongyloides ratti antigen for the detection of $\operatorname{IgG}$ antibodies as confirmatory test in human strongyloidiasis. Mem Inst Oswaldo Cruz 98, 687-691.

Sudarshi, S., Stümpfle, R., Armstrong, M., Ellman, T., Parton, S., Krishnan, P., Chiodini, P. L. \& Whitty, C. J. (2003). Clinical presentation and diagnostic sensitivity of laboratory tests for Strongyloides stercoralis in travellers compared with immigrants in a non-endemic country. Trop Med Int Health 8, 728-732.

ten Hove, R. J., van Esbroeck, M., Vervoort, T., van den Ende, J., van Lieshout, L. \& Verweij, J. J. (2009). Molecular diagnostics of intestinal parasites in returning travellers. Eur J Clin Microbiol Infect Dis 28, 1045-1053.

Verweij, J. J., van Lieshout, L., Blotkamp, C., Brienen, E. A., van Duivenvoorden, S., van Esbroeck, M. \& Polderman, A. M. (2000). Differentiation of Entamoeba histolytica and Entamoeba dispar using PCR-SHELA and comparison of antibody response. Arch Med Res 31, S44-S46.

Verweij, J. J., Canales, M., Polman, K., Ziem, J., Brienen, E. A., Polderman, A. M. \& van Lieshout, L. (2009). Molecular diagnosis of Strongyloides stercoralis in faecal samples using real-time PCR. Trans $R$ Soc Trop Med Hyg 103, 342-346.

Viney, M. E. (1999). Exploiting the life cycle of Strongyloides ratti. Parasitol Today 15, 231-235.

Viney, M. E. \& Lok, J. B. (2007). Strongyloides spp. In WormBook. Edited by The C. elegans Research Community. doi/10.1895/wormbook. 1.141.1, http://www.wormbook.org 